\title{
Bevacizumab for Ovarian Cancer at High Risk of Progression: Reproducibility of Trial Results in 'Real-world' Patients
}

\author{
GIANFILIPPO BERTELLI ${ }^{1}$, FLORIAN DREWS ${ }^{2}$ and KERRYN LUTCHMAN-SINGH ${ }^{2}$ \\ Departments of ${ }^{1}$ Oncology, and ${ }^{2}$ Gynaecological Oncology, Singleton Hospital, Swansea, U.K.
}

\begin{abstract}
Bevacizumab has become a 'community standard' at many UK centres as part of first-line treatment of patients with ovarian cancer at high risk of progression [International Federation of Gynecology and Obstetrics (FIGO) stage IV, or suboptimally debulked stage III] based on the results of phase III trials such as ICON-7. Its impact in patients treated outside clinical trials is, however, still unknown. In this study, we investigated patient characteristics, treatment patterns, adverse events and progression-free survival in 'real-world' patients in South West Wales. A total of 60 patients, treated between 2012 and 2015, were included in the study. Patient characteristics were less favourable compared to the bevacizumab-treated high-risk group in the ICON-7 trial (median age: 66 vs. 60 years; stage IV: $58 \%$ vs. $42 \%$; performance status 0: $18 \%$ vs. $41 \%) ; 75 \%$ had received neoadjuvant chemotherapy before starting bevacizumab. After a median treatment duration of 8 months (range=0-34 months), 45 patients (75\%) had experienced disease progression and 34 (56.7\%) had died. Median progression-free survival was 16 months (95\% confidence interval=14.4-17.6 months). The most common toxicities consisted of proteinuria $(66.7 \%$, all grade $1)$ and grade 1-2 hypertension (15\%). Cardiovascular incidents, two of which were fatal, occurred in $6.7 \%$ of patients. In conclusion, our study provides encouraging evidence that the routine use of bevacizumab as part of firstline treatment of patients with ovarian cancer at high risk of progression may be associated with outcomes comparable with those obtained in clinical trials.
\end{abstract}

Based on the results of two large phase III randomised trials, GOG-0218 and ICON-7 $(1,2)$, bevacizumab was approved by the European Medicines Agency in 2011 for the first-line

Correspondence to: Gianfilippo Bertelli, Singleton Hospital, Sketty Lane, Swansea SA28QA, U.K. E-mail: Gian.bertelli@wales.nhs.uk

Key Words: Ovarian cancer, bevacizumab, adjuvant/neoadjuvant chemotherapy, maintenance treatment, carboplatin and paclitaxel. treatment of advanced ovarian cancer in combination with chemotherapy. A recent update of the ICON-7 trial reported a significant improvement in both progression-free and overall survival associated with bevacizumab in a prespecified high-risk subgroup [International Federation of Gynecology and Obstetrics (FIGO) stage IV, or suboptimally debulked stage III] (3).

In the United Kingdom, although the National Institute for Health and Care Excellence did not recommend its use based on a cost-effectiveness analysis at the licensed dose of $15 \mathrm{mg} / \mathrm{kg}$ (4), bevacizumab at the dose of $7.5 \mathrm{mg} / \mathrm{kg}$ (as in the ICON-7 trial) has become a 'community standard' for oncologists treating ovarian cancer. Patients in England can receive bevacizumab through the Cancer Drug Fund, and local funding arrangements also exist in South West Wales. As a consequence, many patients with ovarian cancer in the UK have bevacizumab incorporated in their first-line treatment; this makes it important to ascertain if this strategy can translate into the expected clinical benefits. Lower chances of response, shorter duration of benefit or increased toxicity cannot be excluded when new treatments are used outside of clinical trials, and it is therefore useful to gather data from patients treated in routine clinical care. 'Realworld' patients often have less favourable characteristics compared to patients eligible for clinical trials, and the incorporation of new treatments in routine clinical practice frequently deviates from the rules followed by trial protocols. For example, the use of neoadjuvant chemotherapy has gained favour in clinical practice based on recent phase III trials $(5,6)$; however, neoadjuvant chemotherapy was not allowed in ICON-7 and GOG-0218 trials $(1,2)$ and was administered to only $13 \%$ of patients participating in GOG0262 trial, a trial which primarily investigated weekly chemotherapy but where $84 \%$ of patients received bevacizumab (7). Thus, the effects of bevacizumab in patients who have received neoadjuvant chemotherapy cannot be reliably predicted based on these trials.

Our study investigated the use of bevacizumab as part of contemporary first-line treatment of advanced ovarian cancer at a single institution in the UK, with the aim of describing 
patient characteristics, treatment patterns, adverse events and progression-free survival.

\section{Patients and Methods}

Consecutive patients with advanced (FIGO stage III-IV) epithelial ovarian, fallopian tube or primary peritoneal cancer, who were managed by the South West Wales Gynaecological Cancer Multidisciplinary Team based in Swansea and received at least one dose of bevacizumab as part of first-line treatment were eligible for this retrospective study. Bevacizumab was given at the dose of 7.5 $\mathrm{mg} / \mathrm{kg}$ every 3 weeks, initially in combination with chemotherapy and then continued as maintenance therapy until disease progression, death or discontinuation due to side-effects. Patients were eligible for bevacizumab if considered at high risk of disease progression, i.e. if they had FIGO stage IV disease, or suboptimally debulked FIGO stage III disease. Suboptimal debulking was defined as any residual disease macroscopically visible after a procedure performed by Gynaecological Oncologists, which was planned to include a midline laparotomy, collection of ascites or washings for cytology, hysterectomy, bilateral salpingoophorectomy and supra/infracolic omentectomy. When appropriate, patients also underwent more radical procedures, including small or large bowel resections with primary anastomosis or stoma formation, gastric resection, cholecystectomy, splenectomy, peritoneal stripping of pelvis, abdomen and/or diaphragm, partial cystectomy with bladder repair or ureteric reimplantation. Debulking surgery was performed either upfront (before starting chemotherapy) or after three or more cycles of neoadjuvant chemotherapy when the initial clinical and radiological presentation predicted a low probability of achieving optimal debulking with upfront surgery in the opinion of the Multidisciplinary Team. Bevacizumab was started with the first or second postoperative chemotherapy cycle, both in patients receiving neoadjuvant chemotherapy and in those undergoing upfront debulking. After completion of chemotherapy, response was assessed radiologically and patients whose disease had not progressed according to Response Evaluation Criteria in Solid Tumors criteria (8) continued with singleagent maintenance bevacizumab every 3 weeks until symptomatic or radiological progression. Asymptomatic cancer antigen 125 (CA125) rise without radiological evidence of progression was not considered a reason for discontinuation of bevacizumab. At the time of progression, patients were offered standard second-line treatment based on their platinum-free interval; bevacizumab was not used again with second-line regimens.

Survival analysis was carried out using SPSS, version 20 (IBM Corp., Armonk, NY, USA). Progression-free survival was determined as the time from the first active treatment (debulking surgery for patients undergoing upfront debulking, or first cycle of chemotherapy for patients receiving neoadjuvant treatment) to the date of progression (radiological or symptomatic) or death (any cause), whichever occurred first. The date of last contact was recorded to censor patients who were still alive and free of progression at the time of the analysis. Due to the relatively short follow-up time, overall survival was not analysed in this study.

\section{Results}

A total of 60 patients with ovarian cancer treated with bevacizumab as part of their first-line chemotherapy between
April 2012 and December 2015 were included in the study. Patient characteristics are summarised in Table I, which also includes a comparison with the bevacizumab-treated highrisk patients in the ICON-7 trial.

Fifteen patients $(25 \%)$ had undergone primary debulking surgery and $45(75 \%)$ interval debulking surgery after three or more cycles of neoadjuvant chemotherapy before starting bevacizumab. After completion of first-line chemotherapy (median of seven cycles, range $=1-10$ cycles), 31 patients (51.7\%) were in complete remission, 23 (38.3\%) had achieved a partial response, five $(8.3 \%)$ had stable disease and response could not be assessed in one patient.

At the time of data analysis (April 2016), patients had received a median of 12 (range $=1-49$ ) doses of bevacizumab, with a median treatment duration of 8 (range $=0-34$ ) months; in 45 patients (75\%) disease had progressed and 34 (56.7\%) had died. The median progression-free survival was 16 months (95\% confidence interval=14.4-17.6 months).

Thirteen patients (21.7\%) were still receiving bevacizumab at the time of analysis, while $47 \mathrm{had}$ discontinued it due to disease progression $(n=35,58.3 \%)$, death without previous documented progression $(\mathrm{n}=3,5 \%)$, toxicity $(\mathrm{n}=7,11.7 \%)$, patient's decision $(\mathrm{n}=1,1.7 \%)$ or clinician's decision $(\mathrm{n}=1,1.7 \%)$; the latter was regarding a woman who was still in complete remission 34 months after starting bevacizumab.

Toxicity was recorded during treatment with bevacizumab and up to 30 days after the last dose, and graded according to Common Terminology Criteria for Adverse Events (CTCAE) version 4.03 (9). Table II summarises toxicity results. The most common toxicities consisted of proteinuria (66.7\%, all grade 1) and grade 1-2 hypertension (15\%). Cardiovascular accidents, two of which were fatal, occurred in $6.7 \%$ of patients. Seven patients $(11.7 \%$ ), whose disease had not yet progressed, discontinued bevacizumab because of side-effects; these included colovesical fistulisation, stroke, haemophtysis, grade 2 hypertension, worsening leg ulcers, elevation of liver function tests, and arthralgia in one patient each. Three patients died without documented progression during treatment with bevacizumab: one died of a myocardial infarction while still in complete remission after 29 months of treatment; the second died of a stroke after the first dose; and the third died of unknown causes after receiving bevacizumab for 8 months.

\section{Discussion}

To our knowledge, this study is the first report of 'realworld' use of bevacizumab in the first-line treatment of patients with high-risk advanced ovarian cancer in the UK. Comparisons of our results with those reported for the highrisk subgroup of the ICON-7 trial must be performed with caution because of the differences in patient characteristics 
Table I. Characteristics of patients treated with bevacizumab for high-risk ovarian cancer in South West Wales (SWW) and in the ICON-7 trial (3).

\begin{tabular}{lcc}
\hline Characteristic & SWW (n=60) & ICON-7 (n=248) \\
\hline $\begin{array}{l}\text { Median age (range), years } \\
\text { FIGO stage, n (\%) }\end{array}$ & $66(37-85)$ & $60(26-80)$ \\
III & $25(42)$ & $144(58)$ \\
IV & $35(58)$ & $104(42)$ \\
WHO PS, n (\%) & & \\
0 & $11(18)$ & $100(41)$ \\
1 & $41(68)$ & $123(50)$ \\
2 & $6(10)$ & $21(9)$ \\
3 & $2(3)$ & 0 \\
Unknown & 0 & $4(2)$ \\
Chemotherapy regimen, n (\%) & $9(15)$ & 0 \\
Carboplatin monotherapy & $51(85)$ & $248(100)$ \\
Carboplatin plus paclitaxel & & \\
\hline
\end{tabular}

FIGO: International Federation of Gynecology and Obstetrics; WHO PS: World Health Organisation performance status.

between the two cohorts. ICON-7 patients were on average younger (median age $60 \mathrm{vs}$. 66 years) and had better performance status (41\% WHO PS $0 v s .18 \%$ ) compared to patients in our study; moreover, fewer patients in ICON-7 had FIGO stage IV disease (42\% vs. 58\%). In ICON-7, all patients received combination chemotherapy with carboplatin and paclitaxel; $15 \%$ of patients in our study were not considered fit enough for combination chemotherapy and were treated with single-agent carboplatin. Despite these differences, that would have predicted worse outcomes in our patients, progression-free survival in our study was encouragingly similar to that reported for bevacizumabtreated high-risk patients in the ICON-7 trial, with a median of 16 months in both cohorts. Of note, the maximum duration of bevacizumab therapy in ICON-7 was 15 months, while patients in our study were allowed to continue maintenance treatment until progression; as a consequence, $16.7 \%$ of our patients received bevacizumab for longer than 15 months.

Taking into account the more advanced age and poor PS of patients in our study, it is not perhaps surprising that four patients $(6.7 \%)$ suffered cardiovascular incidents while receiving bevacizumab, resulting in two possible treatmentrelated deaths (due to stroke and myocardial infarction, respectively). One case of colovesical fistulisation was also observed $(1.7 \%)$, but there was no occurrence of bowel perforation. Hypertension and proteinuria were common but mild and manageable in the majority of patients.

In conclusion, our study provides encouraging evidence that the routine use of bevacizumab as part of first-line treatment of patients with ovarian cancer at high risk of
Table II. Toxicity experienced during treatment with bevacizumab.

\begin{tabular}{lcc}
\hline Toxicity & $\mathrm{n}$ & $\%$ \\
\hline Hypertension & & \\
$\quad$ Grade 1 & 8 & 13.3 \\
$\quad$ Grade 2 & 1 & 1.7 \\
Cardiovascular incidents & & \\
$\quad$ Stroke & 1 & 3.3 \\
Myocardial infarction & 1 & 1.7 \\
Haemophtysis & & 1.7 \\
Other & 40 & \\
Proteinuria & 2 & 66.7 \\
Arthralgia & 1 & 3.3 \\
Infusion site reaction & 1 & 1.7 \\
Colovesical fistula & 1 & 1.7 \\
Increased LFTs & 1 & 1.7 \\
$\quad$ Worsening leg ulceration & & \\
\hline
\end{tabular}

progression may be associated with outcomes comparable with those obtained in clinical trials. Ongoing prospective trials such as ICON8b (where three-weekly carboplatin with paclitaxel and bevacizumab is the 'standard of care' treatment in the control arm) and OSCAR-1 (an observational study gathering 'real-world' data on the use of bevacizumab in the UK) will further elucidate its role.

\section{References}

1 Burger RA, Brady MF, Bookman MA, Fleming GF, Monk BJ, Huang H, Mannel RS, Homesley HD, Fowler J, Greer BE, Boente M, Birrer MJ and Liang SX; Gynecologic Oncology Group. Incorporation of bevacizumab in the primary treatment of ovarian cancer. N Engl J Med 365(26): 2473-83, 2011.

2 Perren TJ, Swart AM, Pfisterer J, Ledermann JA, PujadeLauraine E, Kristensen G, Carey MS, Beale P, Cervantes A, Kurzeder C, du Bois A, Sehouli J, Kimmig R, Stahle A, Collinson F, Essapen S, Gourley C, Lortholary A, Selle F, Mirza MR, Leminen A, Plante M, Stark D, Quian W, Parmar MK and Oza AM; ICON7 Investigators. A phase 3 trial of bevacizumab in ovarian cancer. N Engl J Med 365(26): 2484-2496, 2011.

3 Oza AM, Cook AD, Pfisterer J, Embleton A, Ledermann JA, Pujade-Lauraine E, Kristensen G, Carey MS, Beale P, Cervantes A, Park-Simon TW, Rustin G, Joly F, Mirza MR, Plante M, Quinn M, Poveda A, Jayson GC, Stark D, Swart AM, Farrelly L, Kaplan R, Parmar MK and Perren TJ; ICON7 trial investigators. Standard chemotherapy with or without bevacizumab for women with newly diagnosed ovarian cancer (ICON7): overall survival results of a phase 3 randomised trial. Lancet Oncol 16(8): 928-36, 2015.

4 National Institute for Health and Care Excellence. Bevacizumab in combination with paclitaxel and carboplatin for first-line treatment of advanced ovarian cancer (NICE Technology Appraisal Guidance TA 284), https://www.nice.org.uk/ guidance/ta284 [Accessed 13 June 2016]. 
5 Vergote I, Claes G, Tropé CG, Amant F, Kristensen GB, Ehlen $\mathrm{T}$, Johnson N, Verheijen Rh, van der Burg ME, Lacave AJ, Panici PB, Kenter GG, Casado A, Mendiola C, Coens C, Verleye L, Stuart CG, Pecorelli S and Reed NS; European Organization for Research and Treatment of Cancer-Gynaecological Cancer Group; NCIC Clinical Trials Group. Neoadjuvant chemotherapy or primary surgery in stage IIIC or IV ovarian cancer. N Engl J Med 363(10): 943-953, 2010.

6 Kehoe S, Hook J, Nankivell M, Jayson GC, Kitchener H, Lopes T, Luesley D, Perren T, Bannoo S, Mascarenhas M, Dobbs S, Essapen S, Twigg J, Herod J, McCluggage G, Parmar M and Swart AM. Primary chemotherapy versus primary surgery for newly diagnosed advanced ovarian cancer (CHORUS): an openlabel, randomised, controlled, non-inferiority trial. Lancet 386(9990): 249-57, 2015.

7 Chan JK, Brady MF, Penson RT, Huang H, Birrer MJ, Walker JL, DiSilvestro PA, Rubin SC, Martin LP, Davidson SA, Huh WK, O'Malley DM, Boente MP, Michael H and Monk BJ. Weekly vs. every-3-week paclitaxel and carboplatin for ovarian cancer. N Engl J Med 374(8): 738-48, 2016.
8 Eisenhauer EA, Therasse P, Bogaerts J, Schwartz LH, Sargent D, Ford R, Dancey J, Arbuck S, Gwyther S, Mooney M, Rubinstein L, Shankar L, Dodd L, Kaplan R, Lacombe D and Veweij J. New response evaluation criteria in solid tumours: revised RECIST guideline (version 1.1). Eur J Cancer 45(2): 228-47, 2009.

9 National Cancer Institute: NCI Guidelines for Investigators, Adverse Event Reporting Requirements for DCTD (CTEP and CIP) and DCP INDs and IDEs, http://ctep.cancer.gov/ protocolDevelopment/electronic_applications/adverse_events.ht m [accessed 25 July 2016].
Received July 7, 2016

Revised July 25, 2016

Accepted July 29, 2016 